\title{
Ambiguity Suppression by Azimuth Phase Coding in Multichannel SAR Systems
}

\author{
Federica Bordoni, Marwan Younis, Senior Member, IEEE, and Gerhard Krieger, Senior Member, IEEE
}

\begin{abstract}
The current generation of spaceborne synthetic aperture radar (SAR) systems suffers from a tradeoff between the achievable spatial resolution and swath width. This has motivated intensive research both on more flexible SAR systems, using multiple transmit/receive channels, and on techniques for removing the ambiguities. Among these techniques, the azimuth phase coding (APC), recently proposed to suppress range ambiguities in conventional SAR systems, stands out for its negligible implementation complexity and its effectiveness for point and distributed ambiguities. This paper investigates the possibility of applying the APC technique to the new, forthcoming generation of multichannel SAR systems, based on digital beamforming on receive. The extension of APC to multichannel SAR systems is mathematically described. Specific merit figures are defined to quantify the APC performance. A numerical analysis is developed to characterize the influence on the APC behaviors of the main SAR system parameters. Finally, an example of APC performance is provided, by considering two multichannel SAR systems based on a planar and a reflector antenna.
\end{abstract}

Index Terms-Azimuth phase coding (APC), digital beamforming (DBF), synthetic aperture radar (SAR).

\section{INTRODUCTION}

$\mathbf{T}$ HE LARGE number of recent and forthcoming spaceborne synthetic aperture radar (SAR) missions for remote sensing, e.g., TerraSAR-X, COSMO-SkyMed, RADARSAT-2, TanDEM-X, Sentinel-1, PAZ, testifies the interest that this sector is currently receiving [1]-[5]. Nevertheless, the present generation of systems still suffers a basic limitation in answering to the final user necessities: it does not allow for high spatial resolution and wide coverage simultaneously [6]. For instance, a resolution around $1 \mathrm{~m}$ could be achieved over a swath width of $10 \mathrm{~km}$; whereas a coverage of $100 \mathrm{~km}$ allows for SAR final products with a resolution in the order of $16 \mathrm{~m}$. The importance for many applications of earth observation to overcome this limitation has motivated an intensive research. In particular, since the tradeoff between spatial resolution and swath width is inherent to the system concept and originated by ambiguity constraints, the research has been oriented in two main directions: i) new, more flexible SAR systems [7]-[10]; ii) processing methods for removing the ambiguities from the SAR image [11]-[13].

Manuscript received March 11, 2011; revised May 31, 2011; accepted June 11, 2011. Date of publication September 26, 2011; date of current version January 20, 2012.

The authors are with the Microwaves and Radar Institute, German Aerospace Center (DLR), 82234 Oberpfaffenhofen, Germany (e-mail: federica.bordoni@dlr.de; marwan.younis@dlr.de; gerhard.krieger@dlr.de).

Color versions of one or more of the figures in this paper are available online at http://ieeexplore.ieee.org.

Digital Object Identifier 10.1109/TGRS.2011.2161672
The new generation of SAR systems is mainly characterized by the use of multiple transmit/receive channels and proper digital processing techniques, such as the digital beamforming (DBF) on receive. This allows for a relaxation of the design constraints, a general improvement of the SAR performance, and a mitigation of the tradeoff between swath width and spatial resolution [7], [9], [10], [14].

Among the techniques for ambiguity suppression, the one proposed by Dall and Kusk [13], denoted as azimuth phase coding (APC), has recently received particular interest because of its indiscriminate applicability to point and distributed ambiguities, its low implementation complexity, and the new degrees of freedom that it offers in the system design [13], [15]. The APC is conceived for conventional (one transmit, one receive channel) SAR systems and is based on three main steps: i) azimuth phase modulation on transmission; ii) azimuth phase demodulation on reception; and iii) filtering of the SAR azimuth signal over the processing bandwidth. The modulation and demodulation produce a frequency displacement between the spectrum of the useful signal and that of the range ambiguity. Then, in the presence of Doppler oversampling, i.e., of a gap between the processed bandwidth and the pulse repetition frequency (PRF), the final step iii) allows to filter out the ambiguous signal power, which is located outside the bandwidth of interest [13].

As mentioned, the APC technique proposed by Dall and Kusk is conceived for conventional SAR systems. In [15], the advantages offered by APC, in terms of design and imaging modes, are explained by considering conventional systems and multichannel systems with multiple independent receivers, i.e., imaging multiple subswathes, each according to a conventional method. With respect to these systems, a multichannel system could be characterized by additional digital signal processing. Specifically, multichannel processing techniques are included, to combine the signals received from the different azimuth channels [8], [16]-[18]. This allows for simultaneous wide swath and high-resolution imaging. On the other hand, the properties of the APC are no more guaranteed.

This paper investigates the behaviors and achievable performance of the APC technique, when applied to a multichannel SAR system based on DBF on receive. As an extension to [15], multichannel processing techniques are considered [16]-[18]. The paper is organized as follows. Section II briefly recalls the APC technique in the conventional case. In Section III, the extension of APC to a multichannel SAR system is mathematically described. In Section IV, the parameters are defined, to quantify the ambiguity suppression introduced by the APC. Section $\mathrm{V}$ is dedicated to a numerical analysis, to evaluate the 
performance of APC versus the main system parameters of interest: number of receive channels and oversampling. Then, the APC performance is evaluated by considering two examples of multichannel SAR systems, both based on the highresolution wide-swath (HRWS) specifications and employing a planar array and a reflector antenna, respectively [14], [19], [20]. Further details on the influence of the multichannel processing approach on the APC performance are provided in the Appendix. Finally, the main conclusions are summarized in Section VI.

\section{Review of the Azimuth Phase Coding Technique}

The APC denotes a technique for range ambiguity suppression, conceived for conventional (one transmit, one receive channel) SAR systems [13]. It is based on three main steps:

i) azimuth (i.e., pulse to pulse) phase modulation on transmission;

ii) azimuth phase demodulation on reception;

iii) azimuth filtering over the processing bandwidth.

The modulation/demodulation phases are chosen such that steps i) and ii) cancel each other on the useful signal, which is unchanged by APC; whereas the range ambiguous signals are affected by a residual phase, which depends linearly on the azimuth sample number. As a result, the spectra of the range ambiguities are shifted in the frequency domain and, as they no more superimpose the useful signal, can be filtered out by step iii).

In detail, the APC modulation phase is given by [13]:

$$
\varphi_{\text {mod }}(l)=-\frac{\pi}{M} l^{2}
$$

where $l$ denotes the Tx pulse number; $M \geq 2$ is a positive integer, denoted as APC shift-factor. It is worth noting that for $M=2$, the modulation has a particularly simple form. In fact, taking into account the $2 \pi$ periodicity of $(1), \varphi_{\bmod }(l)$ assumes alternatively the values 0 and $-\pi / 2$.

In up-to-date SAR systems, such as TerraSAR-X, no additional hardware is required to implement the APC modulation. In fact, it can be realized by an offset phase added to transmitted pulses. These pulses can be characterized by using the existing internal calibration schemes. Alternatively, for a phased array antenna, a different antenna excitation with a phase offset can be used. This can be characterized either on-ground or in-orbit by the standard campaigns [21].

The APC demodulation phase is given by [13]:

$$
\varphi_{\text {dem }}(n)=\varphi_{\text {mod }}(n-m)
$$

where $n$ is the receive pulse number (or azimuth sample number); the positive integer $m$ accounts for the round-trip delay of the useful signal, $\tau$, which in satellite systems greatly exceeds the pulse repetition interval: $m / P R F \leq \tau<(m+1) / P R F$. The range ambiguity of order $k$ has an additional roundtrip delay of $k / P R F$ with respect to the useful signal. As a (a)

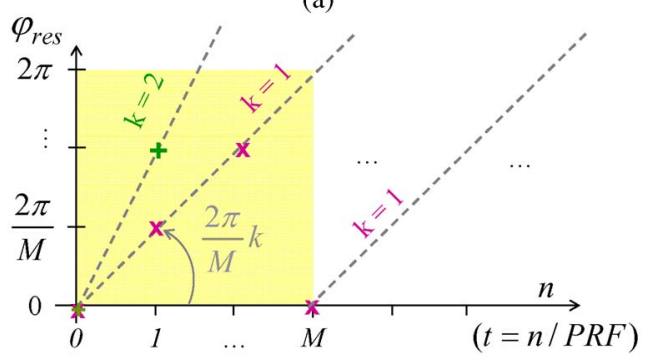

(b)

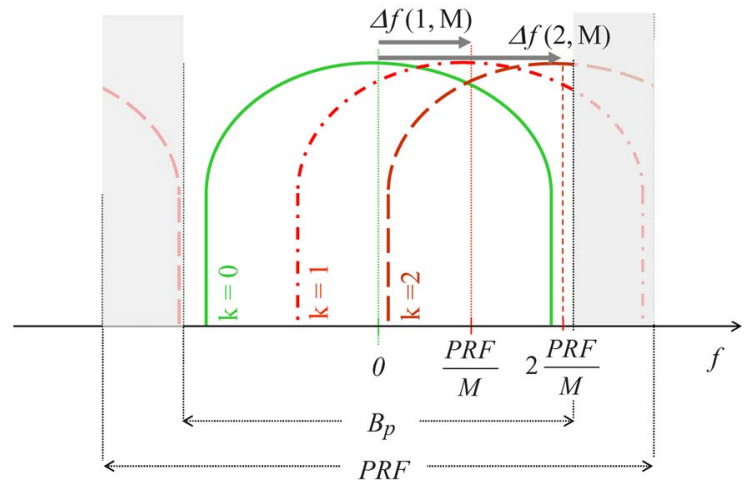

Fig. 1. Application of APC to a conventional SAR system $(M=3)$ : (a) residual phase on the ambiguities of order $k=1,2$. (b) simplified representation of the effect in frequency domain: useful signal (solid) and range ambiguities of order $k=1,2$ (dashed, dash-dotted). The shadowed components can be filtered out.

consequence, after modulation and demodulation, the azimuth signal is affected by the APC residual phase ${ }^{1}[13]$ :

$$
\varphi_{\text {res }}(n, k, M)=\frac{2 \pi}{M} k n, \quad k=0, \pm 1, \pm 2, \ldots
$$

where $k$ is the order of range ambiguity ( $k=0$ for the useful signal). The behaviors of the APC residual phase, $\varphi_{\text {res }}$, versus the sample number $n$, the order of range ambiguity $k$, and the APC shift-factor $M$ are represented in Fig. 1(a). In particular, $\varphi_{\text {res }}$ depends linearly on the azimuth sample number, $n$, with a slope coefficient set by $k$ and $M$ ( $\varphi_{\text {res }}$ can be equivalently represented in $[0,2 \pi)$, where it assumes only $M$ different values). Moreover, $\varphi_{\text {res }}$ has a periodic behavior versus the order of range ambiguity $k$. In fact, an equivalent residual phase affects the ambiguities, whose order differs of an integer multiple of the APC shift-factor $M$; no residual phase, $\varphi_{\text {res }}(n, k)=0$, affects (the useful signal and) the ambiguities, whose order is equal to an integer multiple of $M$. For instance, in case of $M=2$, all the range ambiguities of odd order experience the residual phase, $\varphi_{\text {res }}=0, \pi, 0, \pi, \ldots$; whereas those of even order are unchanged.

In summary, the effect of the APC modulation/demodulation is to multiply the (slow-time) discrete signal, corresponding to the range ambiguity of order $k$, by a phase term, which is linearly dependent on the sample number with a slope, that is set by the ambiguity order itself and the selected factor $M$. In

\footnotetext{
${ }^{1}$ The residual phase includes also an arbitrary constant, which can be neglected [13].
} 
the frequency domain, this corresponds to a shift of the Doppler spectrum of the range ambiguity of order $k$ :

$$
X_{k}(f)=\bar{X}_{k}(f-\Delta f), \quad k=0, \pm 1, \pm 2, \ldots
$$

where $X_{k}(f)$ denotes the discrete fourier transform (DFT) of the range ambiguous signal of order $k$, sampled at the instant $t=n / P R F ; \bar{X}_{k}(f)$ the DFT of the original, i.e., without APC, range ambiguous signal of order $k$;

$$
\Delta f=\Delta f(k, M)=\bmod \left\{k \frac{P R F}{M}\right\}_{P R F / 2}
$$

denotes the APC Doppler shift, set by the order of range ambiguity, $k$, and by the APC shift-factor, $M ; \bmod \{\cdot\}_{P R F / 2}$ the modulus operator, which accounts for the periodicity of the DFT and the limitation of $\Delta f$ within the interval $(-P R F / 2, P R F / 2]$.

Fig. 1(b) outlines the basic effect ${ }^{2}$ of APC in the frequency domain: the APC Doppler shift, $\Delta f$, represents a displacement between the spectrum of the useful signal and that of the ambiguity, which are no more exactly superimposed. As a consequence, the APC final step iii) allows to filter out that part of the ambiguous power, which is located outside the processed bandwidth, $B_{p}$. The larger the frequency displacement, $\Delta f$, between useful signal and range ambiguity with respect to $B_{p}$, the larger the ambiguity component that can be filtered out, i.e., the ambiguity suppression achieved by APC. However, $\Delta f$ cannot be arbitrary large. In fact, the SAR system design is generally performed such that the Doppler oversampling, $P R F / B_{p}$, is limited.

It is worth noting that $M=2$ allows for the best suppression of the range ambiguity of first order, because it maximizes the frequency displacement, $\Delta f=P R F / 2$, between the useful signal and the range ambiguity of first order. Since this ambiguity generally provides the dominant contribute the SAR image quality degradation, the analysis of the APC performance for $M=2$ is particularly significant.

\section{APC In Multichannel SAR Systems BASED ON DigitAl BEAMFORMING ON RECEIVE}

In a conventional SAR system, the application of the APC modulation/demodulation produces a residual linear phase on the range ambiguous signals and consequently a Doppler shift of the range ambiguous spectra. In case of a multichannel SAR system, this is not true anymore.

To explain the previous statement, consider the multichannel SAR system represented in Fig. 2. It employs a single transmit (Tx) and $N$ receive (Rx) azimuth channels. In transmission, the SAR pulse is sent at pulse repetition frequency $P R F$ and a wide azimuth pattern is employed to properly cover, in the Doppler domain, the desired processed bandwidth $B_{p}$. In general, $P R F \ll B_{p}<(N \cdot P R F)$. In reception, on each of the $N$ channels, the azimuth signal is sampled at frequency $P R F$ (i.e., the discrete azimuth signal is represented over a

\footnotetext{
${ }^{2}$ The shape of the spectra is simplified and phenomena such as aliasing and elevation pattern weighting are neglected.
}

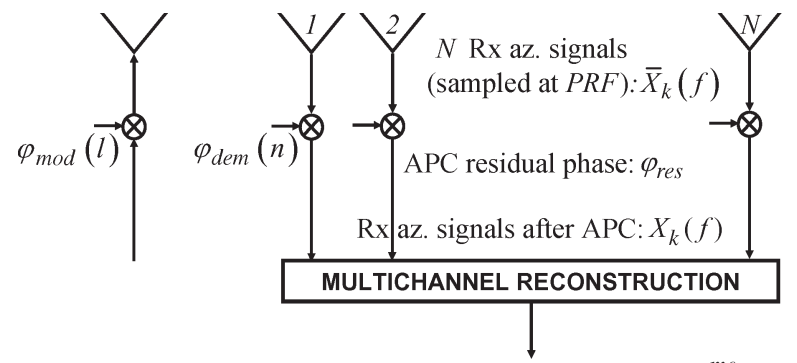

APC residual phase on the reconstructed signal: $\varphi_{\text {res }}^{m c}$ reconstructed multichannel signal sampled at $N P R F: X_{k}^{r}(f)$

Fig. 2. Application of APC in a multichannel SAR system based on DBF on receive.

PRF-width unambiguous frequency interval). Since $P R F \ll$ $B_{p}$, a multichannel processing should be applied to recover the Doppler spectrum over the processed bandwidth $B_{p}$. For instance, in [16], [17], [22], a SAR system based on a planar array antenna is considered, where each receive channel is associated with a single subaperture (i.e., to a wide receive pattern) and to the whole processed bandwidth, $B_{p}$. In this case, the azimuth discrete signal on each receive channel is strongly aliased and the multi-aperture reconstruction algorithm is applied to obtain an unambiguous multichannel signal [16]. Alternatively, in [18], a reflector-based system is considered, where each receive channel is associated with a sharp pattern, covering a subband of the overall processing bandwidth. Here, a proper spectral combination of the signals at each channel allows retrieving the overall band of interest [8].

It is worth remarking that the information necessary to successfully apply the multichannel processing is conveyed by the difference in the acquisition conditions at each $\mathrm{Rx}$ channel. When the APC modulation/demodulation is applied (see Fig. 2), independent on the specific multichannel processing approach, the azimuth signal on each single Rx channel experiences the same residual phases and Doppler shift, given by (3) and (5), as in the conventional SAR case. Since the residual phases at each Rx channel are equal, after multichannel processing, the APC residual phase on the range ambiguous signal has no more a linear trend versus the azimuth sample number, and the properties of APC are no more guaranteed. This assertion is better explained by considering a SAR system based on a planar array antenna, operating with a uniform PRF, ${ }^{3}$ i.e., a PRF matched to the antenna length and number of subapertures such that a regular sampling in azimuth results [17].

In fact, under the hypothesis of uniform PRF, the reconstructed multichannel signal is simply obtained by consecutively interleaving the azimuth samples received by each channel and is equivalent to the Rx signal sampled at the effective PRF, $P R F_{\text {eff }}=N \cdot P R F$ [16], [17], [22]. The APC modulation/demodulation produces the same residual phase, given by (3), on the individual $N$ receivers. Therefore, the residual phase on the reconstructed signal, obtained by interleaving

\footnotetext{
${ }^{3}$ The hypothesis of planar system with uniform PRF allows clarifying more easily the effect of APC in a multichannel system, but does not modify the general conclusions, as shown in the numerical section
} 


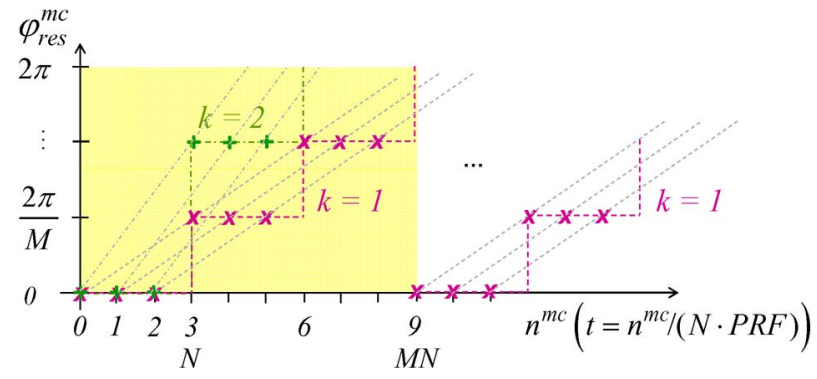

Fig. 3. Application of APC $(M=3)$ to a multichannel SAR system with $N=3 \mathrm{Rx}$ azimuth channels: residual phase on the ambiguities of order $k=1,2$.

the Rx samples, has no more a linear shape but a "stair-shape" (Fig. 3):

$$
\varphi_{r e s}^{m c}\left(n^{m c}, k, M\right)=\frac{2 \pi}{M} k \cdot \text { int }\left\{\frac{n^{m c}}{N}\right\}, \quad k=0, \pm 1, \pm 2, \ldots
$$

where $n^{m c}$ denotes the sample number after multichannel reconstruction (the time interval between succeeding azimuth samples is $\left.1 / P R F_{\text {eff }}\right)$; $\operatorname{int}\{\cdot / N\}$ denotes the integer division by the number of receive channels, i.e., int $\left\{n^{m c} / N\right\}$ is equal to the azimuth sample number $n$ on the single receiver. As in the conventional case, the useful signal is unchanged by $\mathrm{APC}\left(\varphi_{\text {res }}^{m c}(n, 0, M)=0\right)$, and the residual modulation phase is periodic versus the order of range ambiguity $k$ with period $M$.

Then, the azimuth reconstructed signal corresponding to the range ambiguity of order $k$ is:

$$
x_{k}^{r}\left(n^{m c}\right)=\bar{x}_{k}^{r}\left(n^{m c}\right) \exp \left[j \varphi_{\text {res }}^{m c}\left(n^{m c}, k, M\right)\right]
$$

where $\bar{x}_{k}^{r}\left(n^{m c}\right)$ denotes the original (i.e., without APC) azimuth reconstructed signal corresponding to the range ambiguity of order $k$. The DFT of (7) describes the effect of APC in the frequency domain:

$$
X_{k}^{r}(f)=\bar{X}_{k}^{r}(f) \otimes D F T\left\{\exp \left[j \varphi_{r e s}^{m c}(n, k, M)\right]\right\}
$$

where $X_{k}^{r}(f)$ denotes the DFT of $x_{k}^{r}\left(n^{m c}\right) ; \bar{X}_{k}^{r}(f)$ denotes the DFT of $\bar{x}_{k}^{r}\left(n^{m c}\right) ; \otimes$ the convolution operator.

It is worth noting that in real scenarios, the useful signal and the range ambiguity are weighted by different portions of the elevation pattern and are hence subject to different attenuations, because they come from different elevation directions. As a consequence, they have in general a different power (the ambiguity is weaker than the useful signal also without the application of the APC). However, here, the effect of the elevation pattern and geometry is neglected, since the aim of this work is to evaluate the APC performance. In particular, it is assumed that, if the APC were not employed, the signal and the range ambiguity at the input of each receive azimuth channel would have exactly the same spectrum and power, which depends only on the two-way azimuth pattern and phase history. In this sense, it is equivalent to speak of "useful signal" or "original" range ambiguity. (a)

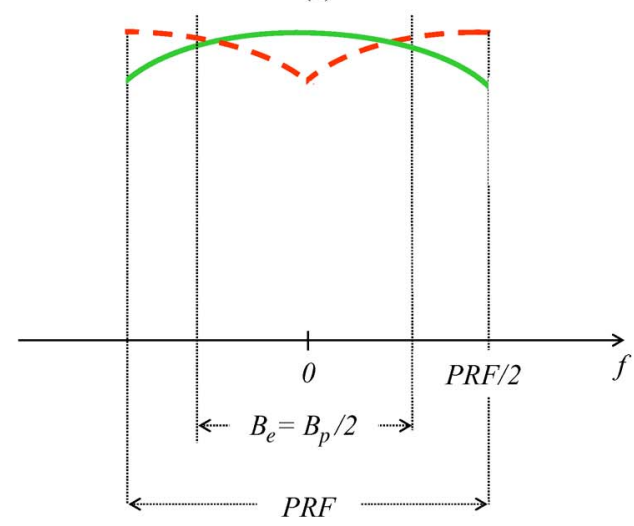

(b)

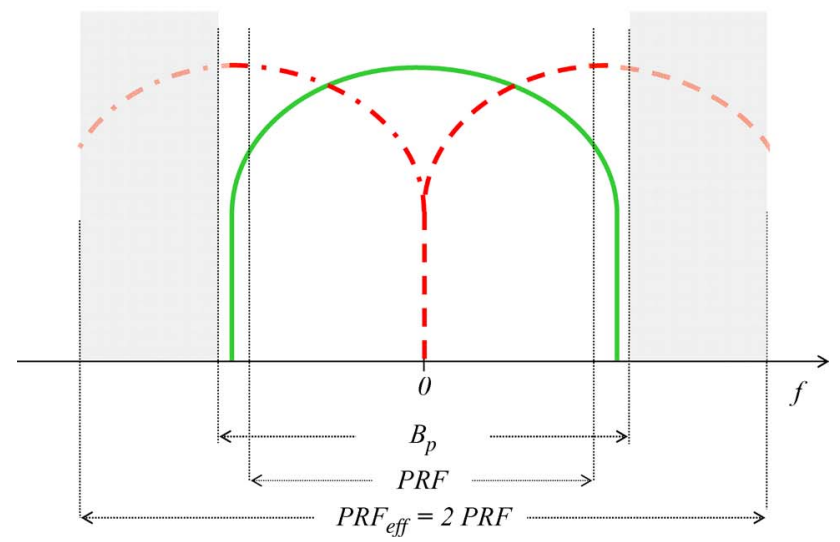

Fig. 4. Application of APC, $M=2$, to a multichannel SAR system, with $N=2 \mathrm{Rx}$ az. channels. Simplified representation of the useful signal (solid) and of the range ambiguities (dashed, dash-dotted) of odd order, in the frequency domain: (a) at single Rx channel level. (b) output of the multichannel reconstruction.

Fig. 4(a) and (b) show a simplified ${ }^{4}$ pictorial representation of the effect of the APC on the received azimuth signal in frequency domain, before (i.e., at single Rx channel level) and after multichannel reconstruction, respectively. The APC shiftfactor $M$ and the number of $\mathrm{Rx}$ azimuth channels $N$ are equal to two. With reference to Fig. 4(b), it is worth noting that, if the APC were applied to an equivalent conventional SAR system operating at $P R F_{\text {eff }}=2 P R F$, the spectrum of the range ambiguities of odd order would be a replica of the useful signal spectrum centered in $P R F$.

\section{Characterization OF THE APC BEHAVIOR AND PERFORMANCE}

To characterize the effect on the SAR signal of the application of the APC technique and the APC performance, two parameters have been defined:

1) APC gain, $G_{a p c}$

2) APC gain at single Rx channel, $G_{a p c}^{s c}$

The APC gain, $G_{a p c}$, provides a measure of the APC range ambiguity suppression and the consequent improvement in terms of SAR image quality. It is measured on the azimuth

\footnotetext{
${ }^{4}$ Effects like aliasing or distortions, induced by the residual phase modulation, are not illustrated for simplicity.
} 
signal, after multichannel processing, as the reduction of power induced by APC on the range ambiguity of first order. In particular, it is given by the ratio between the power of the original (without application of APC) range ambiguity of first order and that of the same ambiguity with application of the APC, computed over the processed bandwidth, $B_{p}$ :

$$
G_{a p c}=\frac{\int_{-B_{p} / 2}^{B_{p} / 2}\left|\bar{X}_{1}^{r}(f)\right|^{2} d f}{\int_{-B_{p} / 2}^{B_{p} / 2}\left|X_{1}^{r}(f)\right|^{2} d f}, \quad B_{p} \leq N P R F
$$

where $\bar{X}_{1}^{r}(f)$ denotes the DFT of the original reconstructed range ambiguity of first order; $X_{1}^{r}(f)$ the DFT of the reconstructed range ambiguity of first order in the presence of the APC. The square module $|\cdot|^{2}$ of the DFT is the power spectral density (PSD) of the corresponding signal.

It is worth remarking that the range ambiguity of first order is generally expected to produce the dominant contribution to the range ambiguity-to-signal ratio (RASR). As a consequence, $G_{a p c}$ provides a measure of the improvement in terms of RASR induced by APC.

The APC gain at single Rx channel, $G_{a p c}^{s c}$, is defined to further characterized the effect of the APC on the SAR signal. It is evaluated before the multichannel processing, at single azimuth Rx channel level..$^{5}$ It is given by the ratio between the power of the original (without application of APC) ambiguous signal of first order and that of the same signal with application of the APC, computed over the equivalent processed bandwidth (EPB), $B_{e}=B_{p} / N$ :

$$
G_{a p c}^{s c}=\frac{\int_{-B_{e} / 2}^{B_{e} / 2}\left|\bar{X}_{1}(f)\right|^{2} d f}{\int_{-B_{e} / 2}^{B_{e} / 2}\left|X_{1}(f)\right|^{2} d f}, \quad B_{e} \leq P R F
$$

where $\bar{X}_{1}(f)$ denotes the DFT of the original range ambiguity of first order; $X_{1}(f)$ the DFT of the range ambiguity of first order, when APC is applied.

The $G_{a p c}$ and $G_{a p c}^{s c}$ are defined also for conventional SAR systems: in this case $N=1, B_{e}=B_{p}$, and $G_{a p c}=G_{a p c}^{s c}$.

\section{NUMERICAl RESUlts}

In this Section, the application of the APC to the forthcoming generation of multichannel spaceborne SAR systems based on DBF on receive is analyzed. First, the APC performance is analyzed versus the main SAR system parameters, which are expected to influence the APC behavior: the number of receive channels and the Doppler oversampling. Then, the APC performance is evaluated for two realistic SAR multichannel systems, based on a planar antenna and a reflector antenna, respectively.

\footnotetext{
${ }^{5}$ In multichannel systems, the APC gain at single Rx channel takes into account only the steps of modulation and demodulation of the APC technique, since evaluated before the multichannel processing.
}

TABLE I

PARAMETERs OF THE PlanAR SAR SySTEMS USED FOR

\begin{tabular}{|c|c|c|c|c|}
\hline Parameter & $\begin{array}{c}\text { System\# } \\
1\end{array}$ & $\begin{array}{l}\text { System\# } \\
2\end{array}$ & $\begin{array}{l}\text { System\#3 } \\
\text { (ref.) }\end{array}$ & $\begin{array}{c}\text { System\# } \\
4\end{array}$ \\
\hline Orbit height & \multicolumn{4}{|c|}{$520 \mathrm{~km}$} \\
\hline Carrier frequency & \multicolumn{4}{|c|}{$9.600 \mathrm{GHz}$} \\
\hline $\begin{array}{l}\text { Tx antenna length, } L_{t x} \\
\text { (and } R x \text { subaperture } \\
\text { length, } L_{r x} \text { sa) }\end{array}$ & \multicolumn{4}{|c|}{$3 \mathrm{~m}$} \\
\hline $\begin{array}{l}\text { Number of azimuth } T x \\
\text { channels }\end{array}$ & \multicolumn{4}{|c|}{1} \\
\hline Rx antenna total length & $3 \mathrm{~m}$ & $6 \mathrm{~m}$ & $12 \mathrm{~m}$ & $24 \mathrm{~m}$ \\
\hline $\begin{array}{l}\text { Number of azimuth } R x \\
\text { channels, } N\end{array}$ & 1 & 2 & 4 & 8 \\
\hline $\begin{array}{l}\text { Pulse repetition } \\
\text { frequency, } P R F\end{array}$ & $5068 \mathrm{~Hz}$ & $2534 \mathrm{~Hz}$ & $1267 \mathrm{~Hz}$ & $633.5 \mathrm{~Hz}$ \\
\hline $\begin{array}{l}\text { Effective pulse } \\
\text { repetition frequency, } \\
\text { PRF } F_{\text {eff }}\end{array}$ & \multicolumn{4}{|c|}{$5068 \mathrm{~Hz}$} \\
\hline
\end{tabular}
PERFoRMANCE ANALYSIS

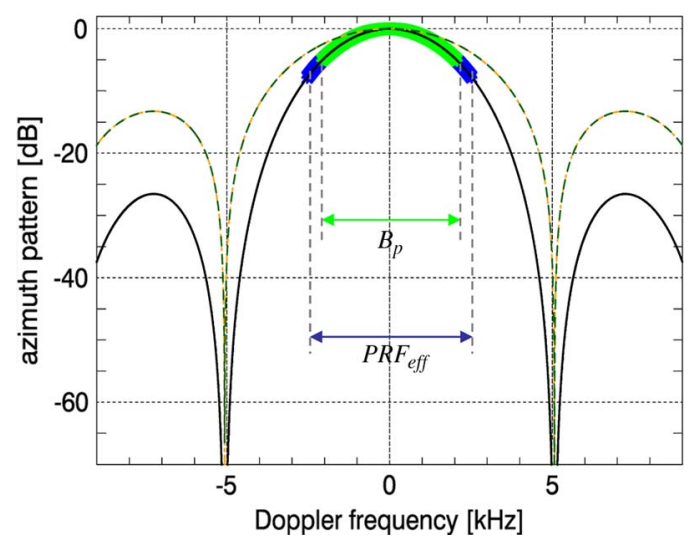

Fig. 5. Azimuth patterns (solid: two-way; dash-dot: Tx; dashed: Rx) of all considered multichannel SAR systems (each azimuth Rx channel is associated with a single azimuth subaperture). An example of processed bandwidth $B_{p}=$ $4168 \mathrm{~Hz}$ and the $P R F_{\text {eff }}=5068 \mathrm{~Hz}$ (the same for all the systems) are shown.

\section{A. Multichannel SAR Systems}

For performance analysis, a reference SAR system based on a planar array antenna with $N=4$ receive subapertures is defined. Its parameters, used for APC performance computation, are summarized in Table I (system \#3).

Moreover, three additional SAR systems are considered, to evaluate the behavior of APC versus the number of $\mathrm{Rx}$ channels, $N$. They are obtained from the reference system by modifying the number of receive subapertures/channels from 4 to 8, 2 and 1, respectively (see systems \#4 and \#2, \#1 in Table I).

The azimuth length of the transmit antenna, $L_{t x}$, and of the receive subapertures, $L_{r x s a}$, is the same for all considered systems. As a consequence, also the Tx and Rx azimuth patterns are identical, as shown in Fig. 5. The multichannel processing is performed according to the multi-aperture reconstruction algorithm, i.e., by assuming that each azimuth $\mathrm{Rx}$ channel is associated with a single subaperture, as in [16], [17] (an alternative multichannel processing approach is described in the Appendix). Therefore, all the considered systems have the same Tx and Rx azimuth patterns. 


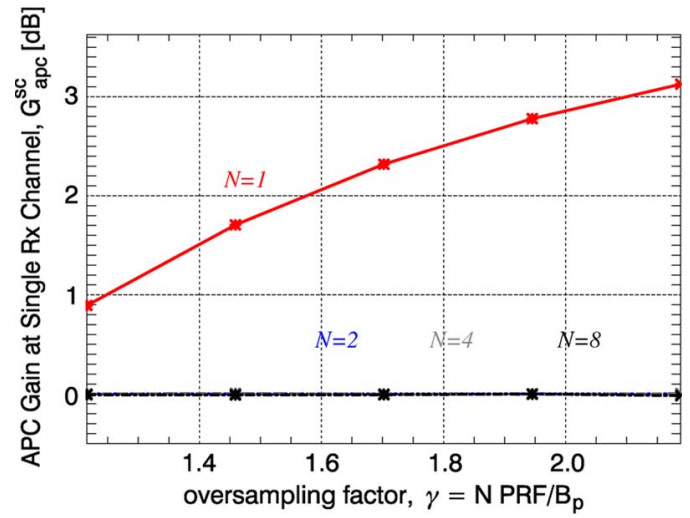

Fig. 6. APC gain at single Rx channel $(M=2)$ versus the oversampling factor, for the SAR systems described in Table I, i.e., for different numbers of receive channels (solid: system \# 1; dotted: \# 2; dashed: \# 3; dash-dot: \# 4).

It is assumed that the SAR systems are operated at uniform PRF:

$$
P R F=\frac{2 \cdot v_{s a t}}{L_{r x s a} \cdot N}=\frac{P R F_{\text {eff }}}{N}
$$

where $v_{\text {sat }}$ denotes the sensor azimuth velocity. The effective PRF, $P R F_{\text {eff }}=2 \cdot v_{s a t} / L_{r x}$ sa , of the reconstructed azimuth signal is the same for all considered SAR systems.

It is reasonable to expect that the performance of the APC depends on the oversampling factor:

$$
\gamma=\frac{P R F_{\text {eff }}}{B_{p}}=\frac{P R F}{B_{e}}=M \frac{\Delta f(1, M)}{B_{e}}
$$

In fact, with reference to the azimuth signal on the single Rx channel (i.e., before the multichannel reconstruction), the oversampling factor is proportional to the Doppler shift introduced by APC on the first order range ambiguity, $\Delta f(1, M)=$ $P R F / M$, normalized to the EPB, $B_{e}$.

To investigate the effect of the oversampling, the APC performance has been evaluated for different processed bandwidth values,

$$
B_{p}=0.89 \frac{v_{\text {beam }}}{\delta_{a z}}
$$

where $v_{\text {beam }}$ is the beam azimuth velocity on ground and $\delta_{a z}$ the azimuth resolution. In particular, the processing bandwidth $2316 \mathrm{~Hz} \leq B_{p} \leq 4168 \mathrm{~Hz}$, which corresponds to an azimuth resolution $0.5 L_{t x} \leq \delta_{a z} \leq 0.9 L_{t x}$ and an oversampling factor $1.2 \leq \gamma \leq 2.2$, has been considered.

As regards the backscattered signal, for performance analysis, the echo backscattered from a point target has been considered.

Fig. 6 shows the APC gain at single Rx channel, $G_{a p c}^{s c}$, versus the oversampling factor, $\gamma=P R F_{\text {eff }} / B_{p}$, obtained for the SAR systems described in Table I, when the APC shift-factor is assumed equal to two $M=2$.

Fig. 6 shows that for the one-channel system (solid line) $0.893 \mathrm{~dB} \leq G_{a p c}^{s c} \leq 3.13 \mathrm{~dB}$, and, as expected, a larger $G_{a p c}^{s c}$ is associated with a larger $\gamma$. In fact, for $N=1$, when the (a)

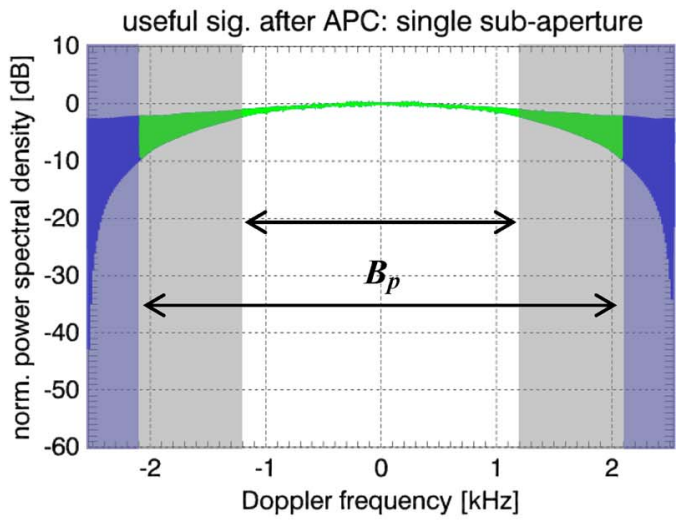

(b)

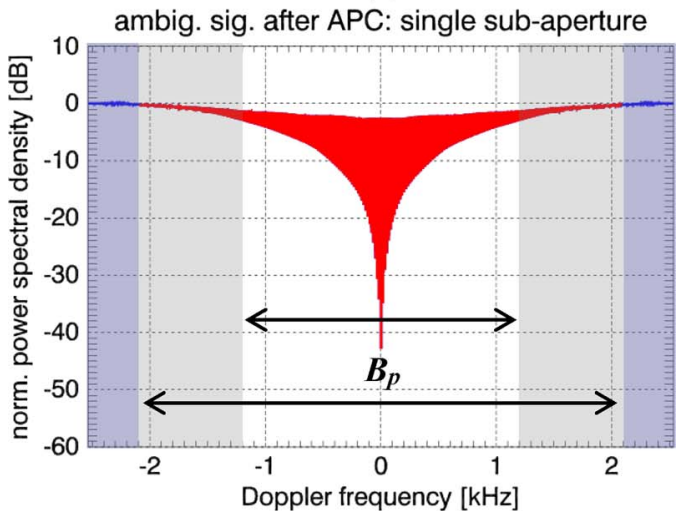

Fig. 7. Computation of the APC gain $\left(G_{a p c}^{s c}=G_{a p c}\right)$ for $N=1$. PSD of a point target over the PRF interval after application of APC $M=2$ : (a) useful signal. (b) first order range ambiguity. The two arrows designate the minimum and maximum processed bandwidths $\left(2316 \mathrm{~Hz} \leq B_{p} \leq 4168 \mathrm{~Hz}\right)$. (The PSD of the useful signal and ambiguity are normalized to the same value, given by the maximum of the PSD of the useful signal.)

oversampling is higher (the APC Doppler shift, $\Delta f$, is constant and the processing bandwidth, $B_{p}$, smaller), a larger part of the spectrum of the first ambiguity falls outside the processing bandwidth and can be filtered out. This is illustrated in Fig. 7, which shows the obtained PSD over the PRF interval of the useful signal (or equivalently the original first order ambiguity) and the first order range ambiguity, for different $B_{p}$ values.

With reference to Fig. 7(a), it is worth noting that the thickness of the PSD curve at the edge of the PRF interval corresponds actually to a fast variation of the spectrum (the apparent compactness is due to the scale of representation). This fast variation is an aliasing effect, produced by the nonfinite Doppler bandwidth of the received echo. (The azimuth ambiguity-to-signal ratio for all the simulated cases varies between $-17 \mathrm{~dB}$ and $-28.5 \mathrm{~dB}$, depending on the selected processed bandwidth.) The PSD in Fig. 7(b) is equal to the PSD in Fig. 7(a) shifted by $\Delta f=P R F / 2$.

Fig. 6 shows also that for the multichannel systems, the $G_{a p c}^{s c}$ is approximately equal to $0 \mathrm{~dB}$. The explanation of this result is that, due to aliasing, the useful signal and the ambiguity have almost the same level over the EPB, $B_{e}=B_{p} / N$. This effect can be seen in Fig. 8, which reports the PSD over the PRF interval (single Rx channel level) of the useful signal and first order ambiguity, for the reference SAR system $(N=4)$. 
(a)

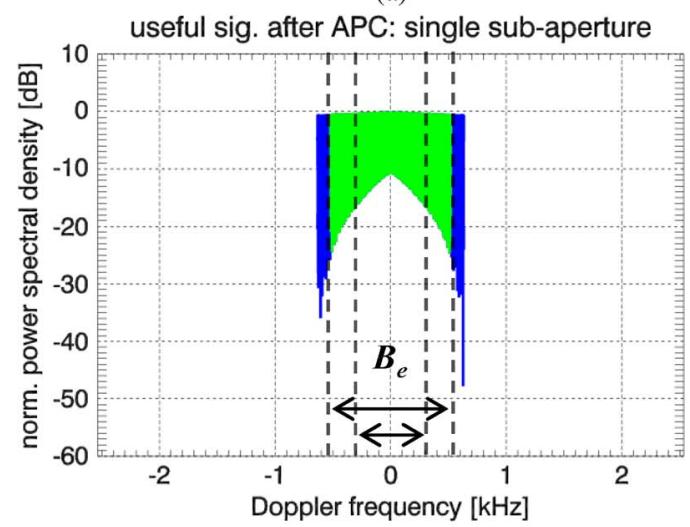

(b)

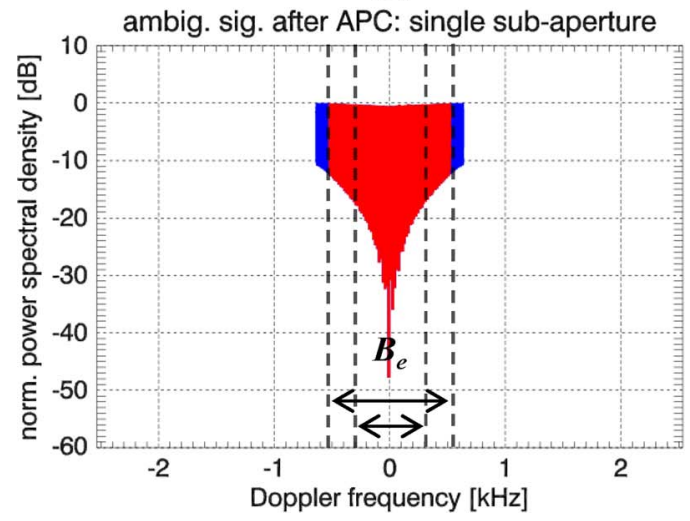

Fig. 8. Computation of $G_{a p c}^{s c}$ for $N=4, P R F=1267 \mathrm{~Hz}$. PSD at single Rx channel level of the signals generated by a point target, $M=2$ : (a) useful signal. (b) first order range ambiguity. The two arrows designate the minimum and maximum equivalent processed bandwidths $\left(579 \mathrm{~Hz} \leq B_{e} \leq 1042 \mathrm{~Hz}\right)$. (The PSD of the useful signal and ambiguity are normalized to the same value, given by the maximum of the PSD of the useful signal.)

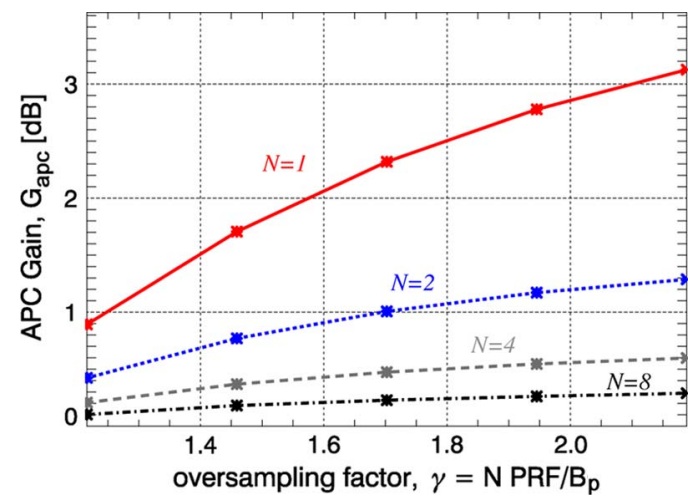

Fig. 9. APC gain $(M=2)$ versus the oversampling factor, for the SAR systems described in Table I, i.e., for different numbers of receive channels (solid: system \# 1; dotted: \# 2; dashed: \# 3; dash-dot: \# 4).

The APC performance, expressed in terms of APC gain, obtained for $M=2$, for the SAR systems in Table $\mathrm{I}$, is represented in Fig. 9 versus the oversampling factor $\gamma=P R F_{\text {eff }} / B_{p}$.

The Figure shows that for the analyzed cases: i) $0.10 \mathrm{~dB} \leq$ $G_{a p c} \leq 3.13 \mathrm{~dB}$; ii) for a given $N$, the $G_{a p c}$ increases with the oversampling factor $\gamma$; iii) the $G_{a p c}$ decreases for increasing number of channels $N$; and iv) the sensitivity of $G_{a p c}$ to $\gamma$ decreases with increasing $N$. (a)

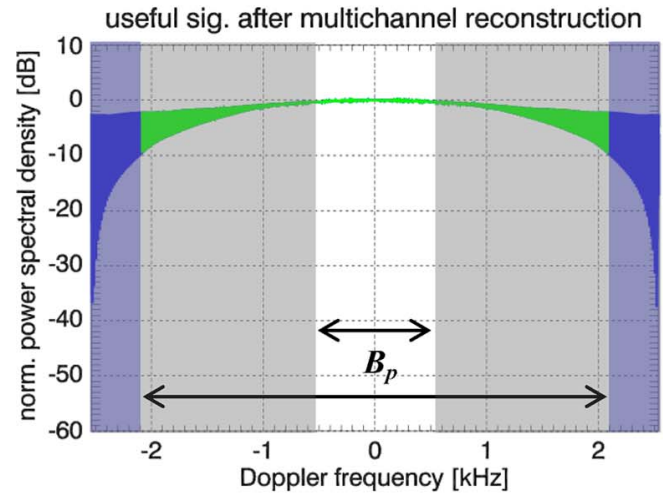

(b)

ambig. sig. after multichannel reconstruction

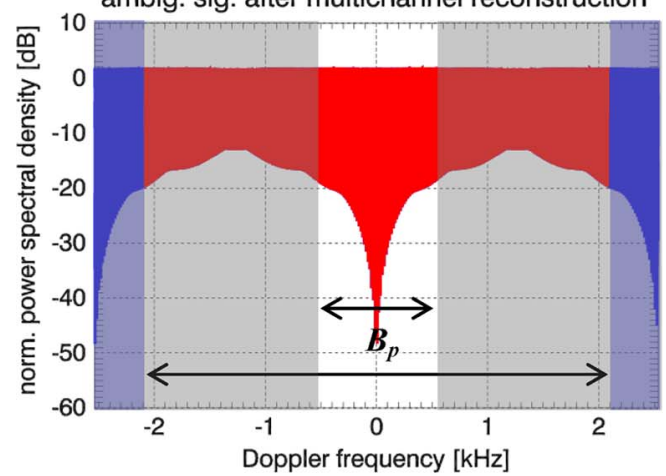

(c)

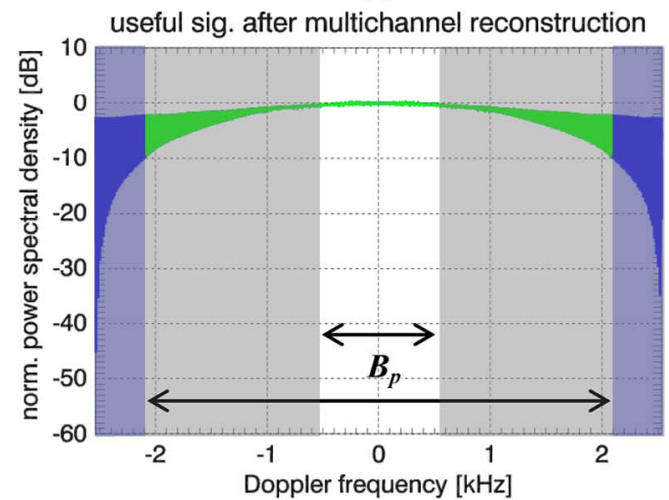

(d)

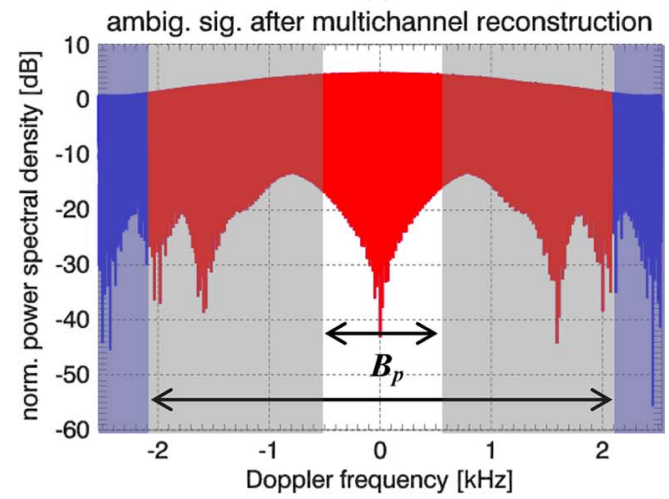

Fig. 10. Computation of $G_{a p c}$. PSD at reconstructed channel level of the signals generated by a point target, $M=2$ : (a) useful signal (or original first order range ambiguity) $N=2$. (b) first order range ambiguity $N=2$. (c) useful signal $N=8$. (d) first order range ambiguity $N=8$. The two arrows designate the minimum and maximum processed bandwidths $(2316 \mathrm{~Hz} \leq$ $B_{p} \leq 4168 \mathrm{~Hz}$ ). (The PSD of the useful signal and ambiguity are normalized to the same value, given by the maximum of the PSD of the useful signal.) 


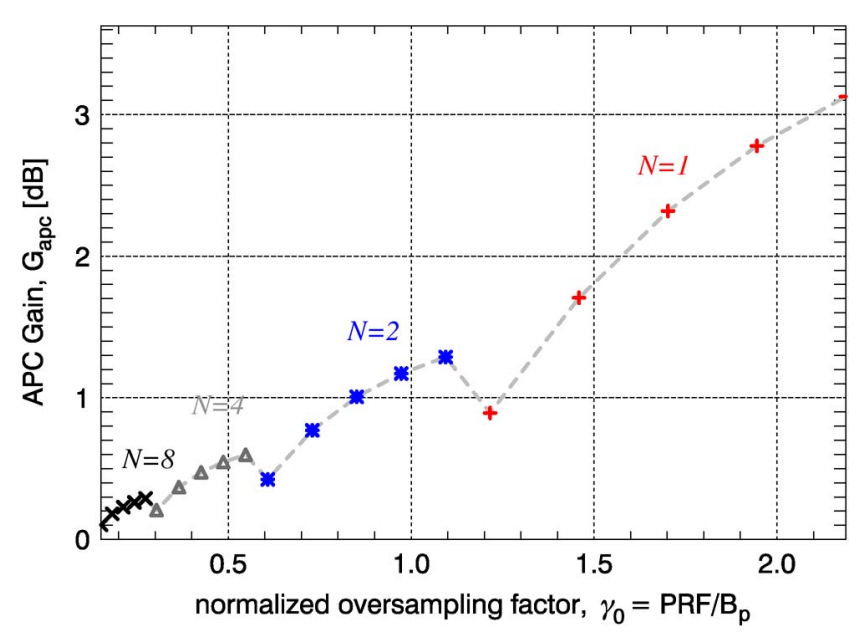

Fig. 11. APC gain versus the normalized oversampling factor, for the SAR systems described in Table I (plus: system \# 1; asterisk: \# 2; triangle: \# 3; X: \# 4).

Fig. 10 shows the PSD involved in the computation of the $G_{a p c}$, in case of $N=2$ and $N=8$. In particular, the PSD is represented over the $P R F_{\text {eff }}$ interval (azimuth reconstructed channel level). For a low number of channels [Fig. 10(a) and (b)], around $f=0 \mathrm{~Hz}$, the useful signal is stronger than the ambiguity, as a consequence a large oversampling $\gamma=$ $P R F_{\text {eff }} / B_{p}$ (i.e., a lower processed bandwidth), produces generally a better $G_{a p c}$. On the other hand, for larger $N$ [Fig. 10(c) and (d)], the upper profile of the PSD of the signal and the ambiguity are similar and consequently $G_{a p c}$ reduces to small values close to $0 \mathrm{~dB}$.

These results are justified by the shape of the residual phase, which implies a spectral spread of the ambiguity, and the decreasing APC Doppler shift of the ambiguity, $\Delta f(1, M)=$ $P R F_{\text {eff }} /(M N)$, for increasing $N$.

It is worth noting that $\gamma$ is independent of the number of receive channels, $N$. To describe the APC performance of a multichannel system, it could be useful to define the parameter $\gamma_{0}$, denoted as the normalized oversampling factor, which conveys the information related to the Doppler oversampling and also to the number of channels, $N$ :

$$
\gamma_{0}=\frac{\gamma}{N}=\frac{P R F}{B_{p}}=\frac{M \Delta f(1, M)}{B_{p}} \propto \frac{1}{N} .
$$

The normalized oversampling, $\gamma_{0}$, is proportional $(\propto)$ to the ratio $\Delta f(1, M) / B_{p}$ of the APC Doppler shift of the first range ambiguity at single Rx channel level, $\Delta f(1, M)=P R F / M$, to the processed bandwidth, $B_{p}$. Moreover, since $B_{p}$ is typically in the order of $N \cdot P R F$, then both $\Delta f / B_{p}$ and $\gamma_{0}$ are inversely proportional to $N$.

The $G_{a p c}$ results, already shown in Fig. 9 versus $\gamma$, are plotted in Fig. 11 versus $\gamma_{0}$.

Fig. 11 shows that the $G_{a p c}$ is almost directly proportional to $\gamma_{0}$ and decreases for increasing number of Rx channels, $N$. The proportionality between $\gamma_{0}$ and $G_{a p c}$ makes the parameter $\gamma_{0}$ suitable to predict the improvement introduced by APC on a given SAR system.
TABLE II

PARAMETERS OF THE HRWS SAR SYSTEM

\begin{tabular}{ll}
\hline \hline \multicolumn{1}{c}{ Parameter } & \multicolumn{1}{c}{ Value } \\
\hline Orbit height & $520 \mathrm{~km}$ \\
Carrier frequency & $9.600 \mathrm{GHz}$ \\
Pulse repetition frequency, PRF & $1750 \mathrm{~Hz}$ \\
Processed bandwidth, $B_{p}$ & $6252 \mathrm{~Hz}$ \\
Equivalent processed bandwidth, $B_{e}$ & $893 \mathrm{~Hz}$ \\
Tx/Rx antenna total length & $8.75 \mathrm{~m}$ \\
Number of azimuth Rx channels, $N$ & 7 \\
Oversampling factor, $\gamma$ & 1.960 \\
Normalized oversampling factor, $\gamma_{0}$ & 0.280 \\
\hline \hline
\end{tabular}

It is worth remarking that the APC performance depends also on the azimuth pattern shape (mainlobe width and ripples, sidelobe level ...). In fact, it is directly involved in the computation of the APC gain and determines the aliasing level. The systems compared in this Section have the same patterns. This allows analyzing the pure effect of the number of Rx channels and of the Doppler oversampling. Nevertheless, it does not help clarifying the influence of the pattern on the APC performance. The effect of the pattern on the APC performance is exemplified in the following paragraphs, by considering two real multichannel SAR systems.

\section{B. HRWS SAR System Based on a Planar Antenna}

In this Section, the APC performance is evaluated by considering the HRWS SAR system, the multichannel system, promoted by the German Aerospace Centre (DLR), conceived to obtain simultaneously high-resolution and wide coverage [14], [19], [23], [24].

The HRWS SAR system is based on the SCan-On-REceive algorithm [14]: a large swath is illuminated by using a wide, static transmit beam; whereas in reception, DBF is employed to obtain a sharp and high gain pattern, which follows the pulse echo travelling along the ground swath. The conflict between swath width and azimuth resolution is overcome by using multiple phase centers located along the azimuth direction and the application of the multi-aperture reconstruction algorithm [16].

The main parameters of the system are summarized in Table II [19]. As shown in the Table, the HRWS is a monostatic system, based on a planar antenna. The azimuth patterns are shown in Fig. 12. In particular, in transmission, a wide pattern is obtained by using the whole aperture and the phase spoiling technique; in reception, each channel is associated with the uniform pattern of a single subaperture [19]. This system achieves a spatial resolution of $1 \mathrm{~m}$ and a swath width of $70 \mathrm{~km}$ in basic stripmap mode [19].

The APC gain values obtained for $M=2$ are: $G_{a p c}=$ $0.686 \mathrm{~dB}$ and $G_{a p c}^{s c}=-0.072 \mathrm{~dB}$; the PSD involved in the computation are shown in Fig. 13. The HRWS system, based on a planar antenna with $N=7$ receive channels, has a normalized oversampling factor $\gamma_{0}=P R F / B_{p}=0.28$. As a consequence, the aliasing level is high on the single $\mathrm{Rx}$ channel. This explains why $G_{a p c}^{s c}$ is approximately equal to $0 \mathrm{~dB}$. In 


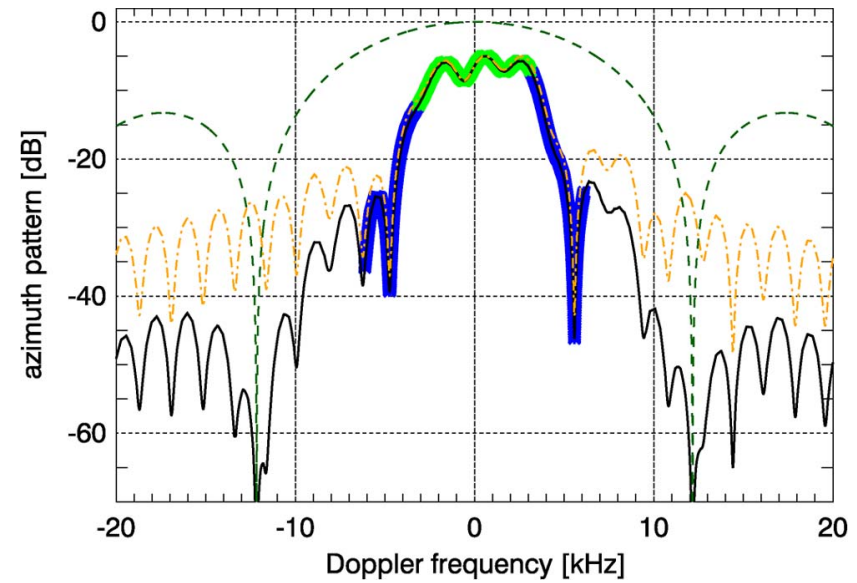

Fig. 12. Azimuth patterns of the HRWS SAR system in Table II (solid: twoway; dash-dot: Tx; dashed: Rx). The $B_{p}$ and $P R F_{\text {eff }}$ intervals are marked by a fat line on the two-way pattern.

fact, the signal power is almost uniformly distributed over the unambiguous frequency interval $(-P R F / 2, P R F / 2$ ] [see Fig. 13(a) and (b)].

Moreover, as expected, the high number of channels is associated with a low $G_{a p c}$. In fact, it corresponds to small values of $\gamma_{0}$ and $\Delta f / B_{p}$ (for this system, $\Delta f=P R F / 2=$ $875 \mathrm{~Hz} \ll B_{p}=6252 \mathrm{~Hz}$ ). Consequently, after multichannel reconstruction, also the difference between the spectrum of the signal and of the ambiguity is small [see Fig. 13(c) and (d)].

\section{HRWS SAR System Based on a Reflector Antenna}

In this Section, the APC is applied to an alternative design option of the HRWS, currently studied at the German Aerospace Centre (DLR) [18], [20], [25]. It is based on a reflector antenna and characterized by the same SAR performance requirements as the planar antenna HRWS system, described in the previous Section. In particular, it achieves a spatial resolution of $1 \mathrm{~m}$ and a swath width of $70 \mathrm{~km}$ in stripmap mode. The main system parameters are summarized in Table III.

With respect to the planar antenna-based system, in the reflector-based system, a different strategy is used to process the multichannel signal [18], [20], [25]. In particular, as for the planar case, a wide Tx azimuth pattern is designed to cover the whole processing bandwidth, $B_{p}$. Nevertheless, in reception, each azimuth channel is associated with a sharper pattern, covering in a bandpass way a specific Doppler subband, $B_{p, i}$ with $i=1,2, \ldots, N$. These Doppler subbands have the same width, approximately equal to $B_{e}=B_{p} / N$ and cover together the whole processing bandwidth:

$$
B_{p}=B_{p, 1} \cup B_{p, 2} \cup \ldots \cup B_{p, i} \cup \ldots \cup B_{p, N} .
$$

Fig. 14(a) shows an example of azimuth patterns. It is worth noting that the azimuth pattern shape depends on the range position, since azimuth and elevation patterns of a reflector system are not independent of each other [18], [20], [25]. (a)

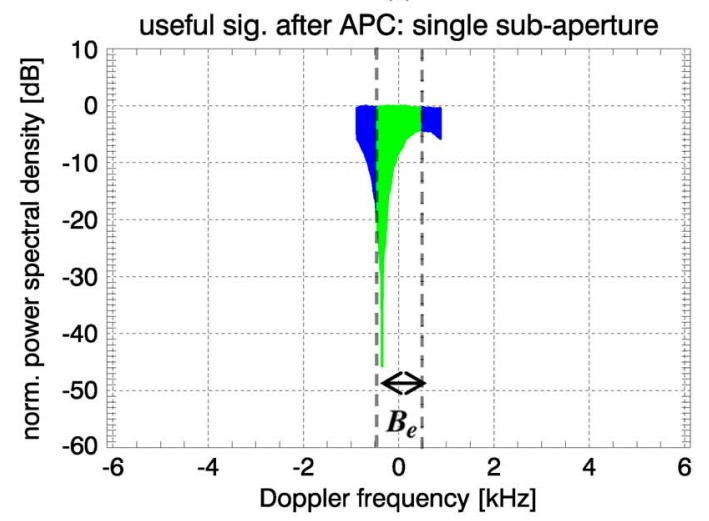

(b)

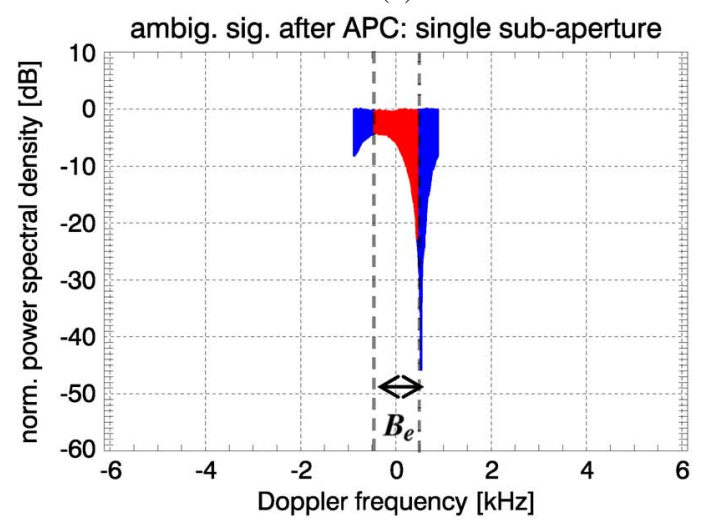

(c)

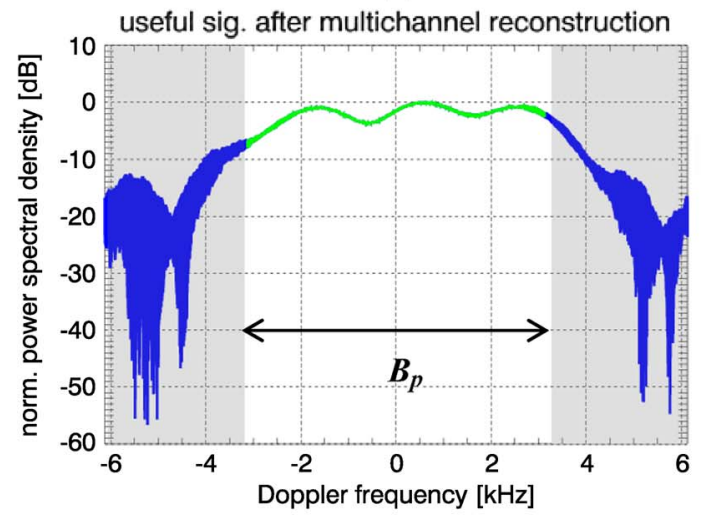

(d)

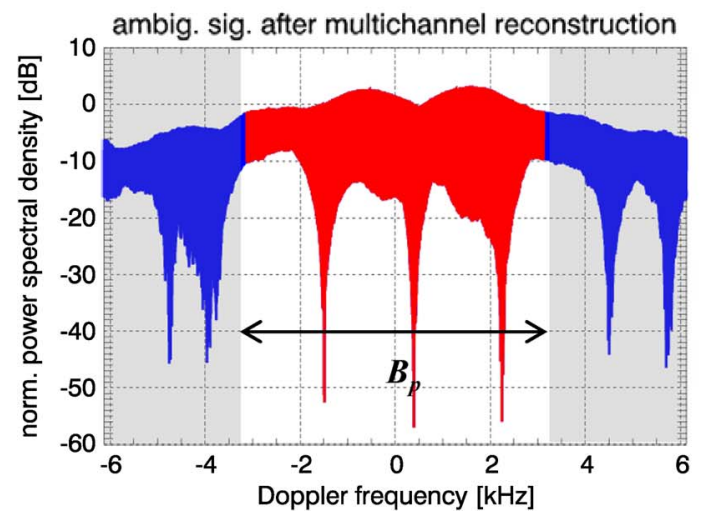

Fig. 13. Azimuth patterns of the HRWS SAR system in Table II (solid: twoway; dash-dot: Tx; dashed: Rx). The $B_{p}$ and $P R F_{\text {eff }}$ intervals are marked by a fat line on the two-way pattern. (a) single Rx channel, useful signal. (b) single Rx channel, first range ambiguity. (c) after multichannel reconstruction, useful signal. (d) after multichannel reconstruction, first range ambiguity. 
TABLE III

PARAMETERS OF THE REFLECTOR-BASED HRWS SAR SySTEM

\begin{tabular}{ll}
\hline \hline \multicolumn{1}{c}{ Parameter } & \multicolumn{1}{c}{ Value } \\
\hline Orbit height & $745 \mathrm{~km}$ \\
Carrier frequency & $9.650 \mathrm{GHz}$ \\
Pulse repetition frequency, PRF & $2792 \mathrm{~Hz}$ \\
Processed bandwidth, $B_{p}$ & $5946 \mathrm{~Hz}$ \\
Equivalent processed bandwidth, $B_{e}$ & $594.6 \mathrm{~Hz}$ \\
Paraboloid diameter (elevation, azimuth) & $10 \mathrm{~m}, 12 \mathrm{~m}$ \\
Total number of feeds (elevation, azimuth) & 60,10 \\
Number of azimuth Rx channels, $N$ & 10 \\
Oversampling factor, $\gamma$ & 4.696 \\
Normalized oversampling factor, $\gamma_{0}$ & 0.4696 \\
\hline \hline
\end{tabular}

(a)

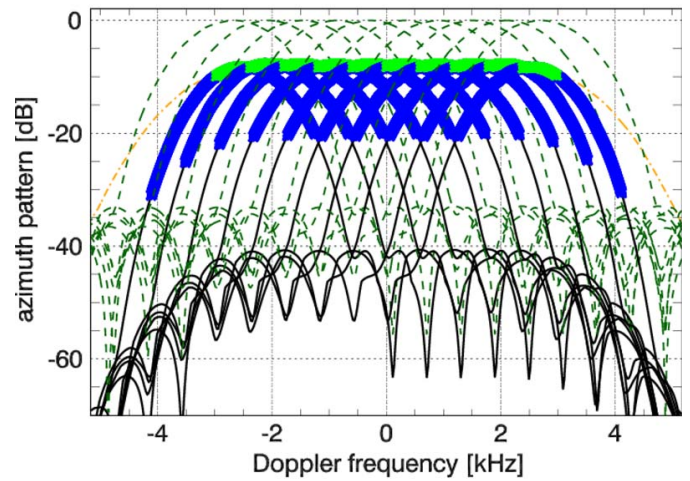

(b)

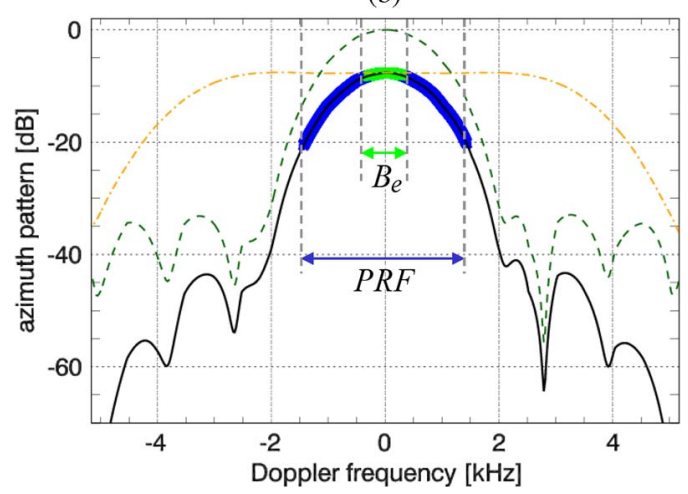

Fig. 14. Example of azimuth patterns of the reflector-based HRWS SAR system (solid: two-way; dash-dot: Tx; dashed: Rx): (a) all Rx patterns with squint. (b) single Rx pattern, with no squint. The $B_{e}$ and $P R F$ intervals are marked by a fat line on the two-way patterns.

The multichannel processing method, based on multirate and DBF processing, could be interpreted as a spectral apposition of the Rx channel contributes over the processed bandwidth, $B_{p}$, (i.e., as a side by side arrangement of the signals at each $\mathrm{Rx}$ channel, made in the frequency domain according to subband associated with the channel) [8], [18].

The different multichannel processing approach affects the computation of the APC gains. In particular, for the reflectorbased system (and more in general for multichannel systems using this multichannel processing approach ${ }^{6}$ ), the $G_{a p c}^{s c}$ and

\footnotetext{
${ }^{6}$ In a planar antenna-based system the multichannel reconstruction could be performed according to this approach as well (see Appendix) [8].
}

$G_{a p c}$ have approximately the same value and can be computed on the single azimuth channel as (see Fig. 14): ${ }^{7}$

$$
\begin{aligned}
G_{a p c} \cong G_{a p c}^{s c} \cong \frac{\left[\int_{B_{p, i}}\left|\bar{X}_{1}(f)\right|^{2} d f\right]}{\left[\int_{B_{p, i}}\left|X_{1}(f)\right|^{2} d f\right]} \\
\simeq \frac{\left[\int_{-B_{e} / 2}^{B_{e} / 2}\left|\bar{X}_{1}(f)\right|^{2} d f\right]}{\left[\int_{-B_{e} / 2}^{B_{e} / 2}\left|X_{1}(f)\right|^{2} d f\right]}, \quad i=1,2, \ldots, N
\end{aligned}
$$

where $B_{p, i}$ denotes the Doppler subband associated with the $i$ th channel; $\bar{X}_{1}(f)$ the DFT of the original range ambiguity of first order; $X_{1}(f)$ the DFT of the range ambiguity of first order, when APC is applied. In the numerical analysis, for the computation of the APC gain in (16), the squint of the Rx azimuth pattern associated with the single subaperture is neglected: the maximum of the Rx pattern is assumed in the zero Doppler, and the APC is computed over the band $\left[-B_{e} / 2, B_{e} / 2\right]$ [see Fig. 14(b)].

The obtained APC gain value changes along the swath, due to the variation of the pattern shape [18], [20], [25]: $3.22 \mathrm{~dB} \leq$ $G_{a p c} \leq 8.60 \mathrm{~dB}$, for $M=2$. The APC gain variation demonstrates the important influence on the APC performance of the azimuth pattern shape. In particular, it proves that the APC gain can be considerably different in two SAR systems, characterized by the same number of Rx channels and Doppler oversampling but by different patterns.

The PSD involved in the computation, for the best APC performance case along the swath, is shown in Fig. 15 (the azimuth patterns in Fig. 14 refer also to this case).

The good APC performance of the reflector-based HRWS system with respect to that of the planar system is justified by the higher oversampling and the more favorable azimuth pattern shape: $\gamma=N P R F / B_{p}=4.7, \gamma_{0}=P R F / B_{p}=0.47$ (the corresponding parameters for the HRWS planar system are $\gamma=1.960$ and $\gamma_{0}=0.280$ ); the pattern sidelobe level is in the order of $-25 \mathrm{~dB}$ and $-35 \mathrm{~dB}$ in the worst and best performance case along the swath, respectively (for the planar HRWS system it is about $-20 \mathrm{~dB}$ ).

In general, the APC performance of the reflector-based system is expected to be better than that of the planar antennabased system providing the same SAR performance, because the reflector-based systems are usually characterized by a higher oversampling. In fact, a high oversampling is used in reflector systems to reduce the azimuth ambiguities resulting from the degradation of azimuth patterns at the swath edges [25]. As a consequence, reflector-based systems can take better advantage from the application of the APC.

\footnotetext{
${ }^{7}$ It is assumed that the differences between the two-way patterns at each azimuth channel are negligible.
} 
(a)

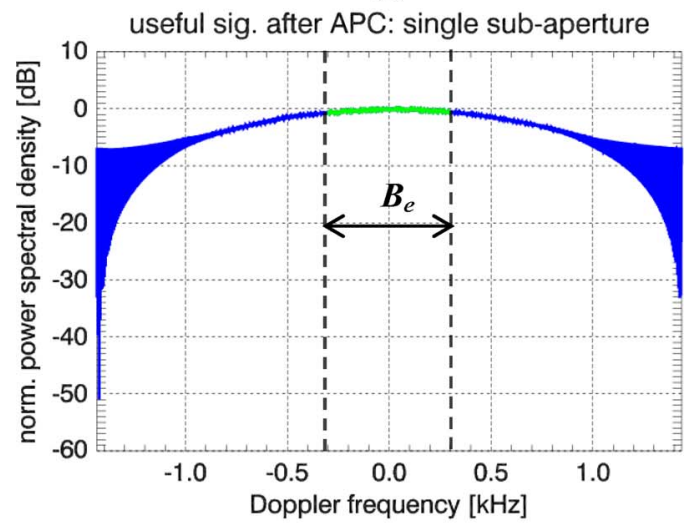

(b)

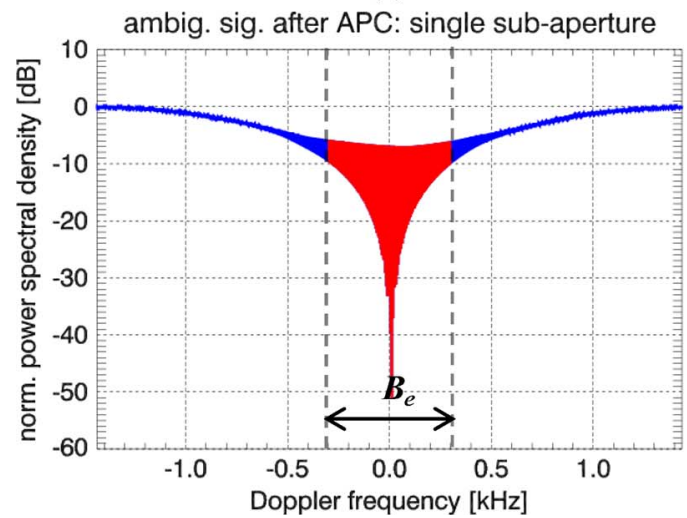

Fig. 15. HRWS SAR system based on a reflector antenna; azimuth signals backscattered from a point target; $M=2$ : (a) PSD of the useful signal on the single Rx channel. (b) PSD of the first order range ambiguity on the single Rx channel. Best results over the swath. The arrow designates equivalent processed bandwidth. (The useful signal and ambiguity PSD are normalized to the maximum of the useful signal PSD.)

\section{CONCLUSION}

The possibility to apply the APC technique to multichannel SAR systems based on DBF on receive has been investigated. The achievable performance of the APC technique in conventional and multichannel systems has been evaluated.

In particular, a mathematical description of the effect of the multichannel processing on the APC properties has been derived. The reported analysis demonstrates that the multichannel processing affects the APC properties. In fact, it is still true that the APC produces a residual phase on the SAR signal; that this phase depends on the order of range ambiguity and is zero for the useful signal. Nevertheless, the expression of the APC residual phase changes: it has not a linear trend versus the sample number. Consequently, the APC effect cannot be represented by a simple frequency shift of the range ambiguity. Based on this analysis, specific parameters have been defined, to quantify the APC ambiguity suppression. Then, a numerical investigation has been developed, to examine the dependence of the APC performance on the SAR system parameters, number of channels and oversampling. This investigation has been further extended by considering two multichannel SAR systems, utilizing a planar and a reflector antenna.

The obtained results show that also in the multichannel case, the application of the APC technique allows for improved

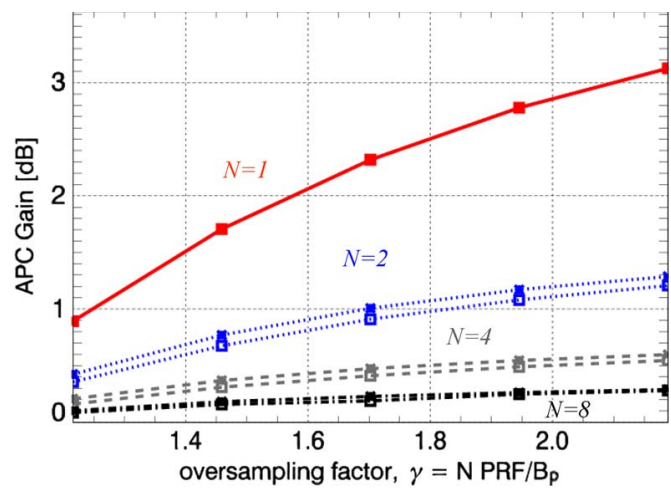

Fig. A.1. APC $(M=2)$ performance for the SAR systems described in Table I (solid: system \# 1; dotted: \# 2; dashed: \# 3; dash-dot: \# 4): Gapc (asterisk symbol) and $G_{a p c}^{T L}$ (square symbol).

ambiguity suppression. In more detail, an important role on the achievable APC performance is played by the two-way azimuth pattern shape. For a given pattern, the ambiguity suppression achieved by APC is directly proportional to the oversampling factor, $\gamma=N P R F / B_{p}$, and inversely proportional to the number of receive channels $N$. In a concise way, it is roughly proportional to the so-called normalized oversampling factor, $\gamma_{0}=P R F / B_{p}$.

A large oversampling does not typically occur in multichannel systems based on a planar antenna architecture, but could be achieved in reflector-based systems. As a consequence, the use of the APC technique is recommended in reflector-based systems. For instance, in the analyzed reflector-based system, characterized by a $\gamma_{0}=0.47$ and an azimuth pattern sidelobe level between $-25 \mathrm{~dB}$ and $-35 \mathrm{~dB}$, the suppression of each ambiguity of odd order reaches values between $3 \mathrm{~dB}$ and $8 \mathrm{~dB}$. In planar array systems, the expected improvement introduced by APC is generally smaller. For instance, in the planar antenna HRWS system, characterized by a $\gamma_{0}=0.28$ and an azimuth pattern sidelobe level of $-20 \mathrm{~dB}$, it is about $0.7 \mathrm{~dB}$. However, regardless of the actual ambiguity suppression, it could be reasonable to recur to APC, given the low implementation complexity of the technique and the increased flexibility in the SAR system design that it offers. Finally, it is worth remarking that APC can be effectively utilized in SAR systems for high-resolution and wide swath imaging, operating with a single azimuth but multiple elevation channels. Such systems simultaneously image multiple (up to 20) swathes and employs a high PRF to achieve the required azimuth resolution [8]. Here, the APC may become extremely attractive because range ambiguities are the system performance driver and they could be significantly attenuated through APC, working with a high oversampling.

\section{APPENDIX}

In this Section, the APC gain in planar systems is evaluated by assuming that the multichannel processing is performed according to the approach based on multirate and DBF processing, considered so far only for the reflector system [8], [18]. According to this approach, in transmission, a wide 
(a)

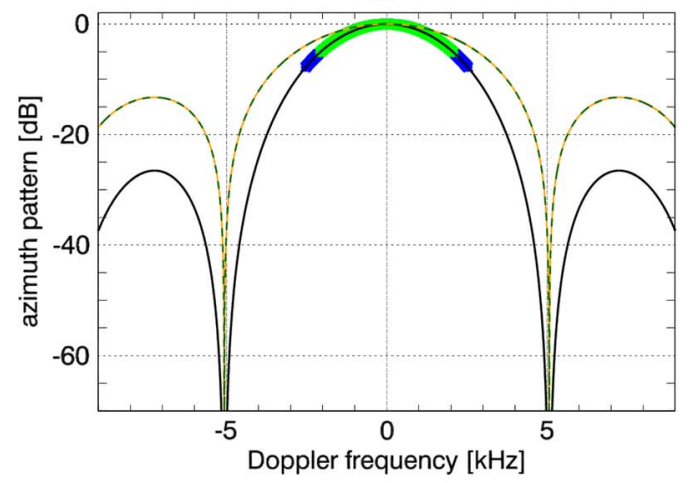

(b)

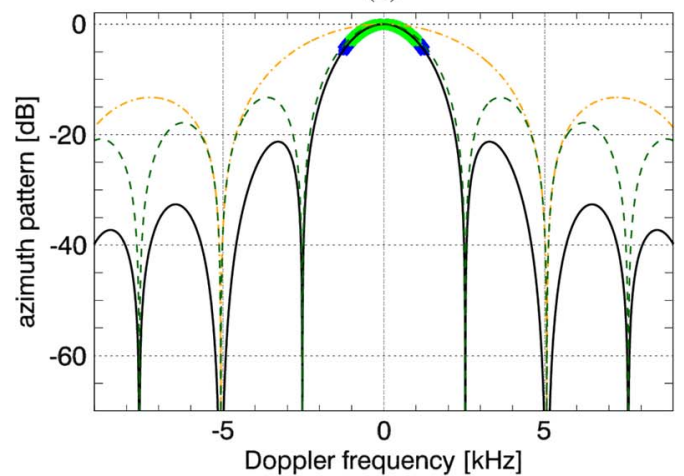

(c)

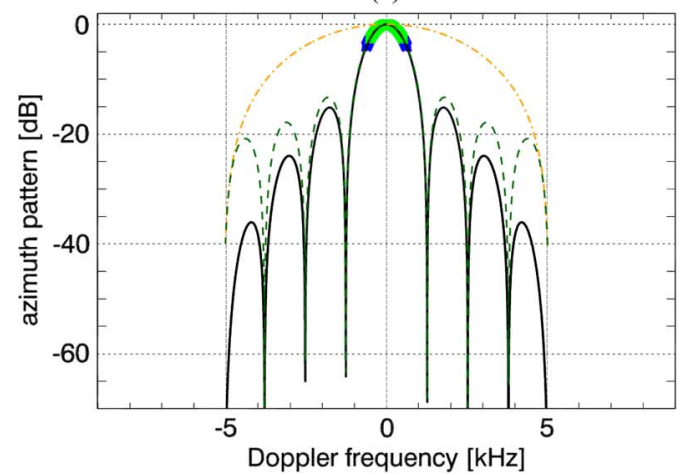

Fig. A.2. Azimuth pattern (solid: two-way; dash-dotted: Tx; dashed: Rx) of: (a) the system \#1 in the TL operational mode $(N=1)$. (b) the system \#2 in the TL operational mode $(N=2)$. (c) the system \#3 in the TL operational mode $(N=4)$. The $B_{e}$ and PRF intervals are marked by a fat line on the two-way pattern.

azimuth pattern illuminates the whole processing bandwidth, $B_{p}$; whereas in reception, each of the $N \mathrm{Rx}$ azimuth channel is associated with a sharper pattern and a specific Doppler subband, $B_{p, i}$, as in (15). In particular, let us assume that each Rx channel is associated with the total azimuth receive aperture [8]. Then, as in the reflector case, the APC gain can be measured at single Rx channel level. In particular, by using (16), it is given by:

$$
G_{a p c}^{T L} \cong \frac{\left[\int_{-B_{e} / 2}^{B_{e} / 2}\left|X_{1}^{T L}(f)\right|^{2} d f\right]}{\left[\int_{-B_{e} / 2}^{B_{e} / 2}\left|X_{1}^{T L}(f)\right|^{2} d f\right]}, \quad i=1,2, \ldots, N
$$

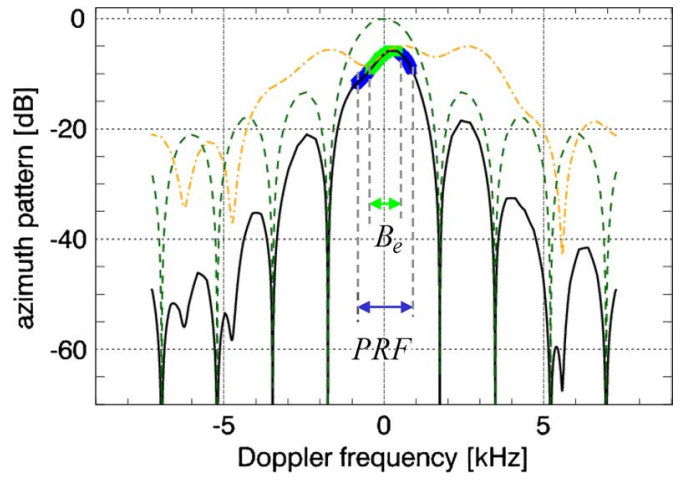

Fig. A.3. Azimuth pattern (solid: two-way; dash-dotted: Tx; dashed: Rx) of the planar HRWS system. The $B_{e}$ and $P R F$ intervals are marked by a fat line on the two-way pattern.

where $\bar{X}_{1}^{T L}(f)$ denotes the DFT of the original range ambiguity of first order; $X_{1}^{T L}(f)$ the DFT of the range ambiguity of first order, when APC is applied; the apex "TL" is used to recall that the corresponding quantity is obtained by using the total length (TL) of the Rx aperture.

Fig. A. 1 represents the APC performance, in terms of $G_{a p c}$ and $G_{a p c}^{T L}$, of the planar systems considered in Section V-A. It shows that the $G_{a p c}^{T L}$ is similar and slightly smaller with respect to the corresponding $G_{a p c}$ value.

The previous results can be explained by looking at Fig. A.2, which shows the azimuth patterns of the systems considered in Section V-A with $N=1,2,4 \mathrm{Rx}$ azimuth channels. In the TL case, the azimuth receive pattern is no more independent on number of Rx channels, but became sharper in the mainlobe as $N$ increases. Accordingly, the ratio between the two-way pattern mainlobe width and the bandwidth of interest for the computation of $G_{a p c}^{T L}$ and $G_{a p c}\left(B_{e}=B_{p} / N\right.$ for $G_{a p c}^{T L}, B_{p}$ for $\left.G_{a p c}\right)$ is similar, though a little more disadvantageous in the TL case (since only the Rx pattern is different, the two-way pattern is proportionally wider in the computation of $G_{a p c}^{T L}$ than in $\left.G_{a p c}\right)$.

For the planar HRWS system described in Section V-B, $G_{a p c}^{T L}=1.065=0.275 \mathrm{~dB}$ and $G_{a p c}=1.171=0.686 \mathrm{~dB}$. In this case, with respect to the previous results, the difference between the two APC gain values is larger. This is mainly due to the irregularity of the HRWS azimuth Tx pattern over the processed bandwidth (see Figs. 12 and A.3), which affects the approximation in (A.1) and makes the $G_{a p c}^{T L}$ value slightly dependent on the subband of computation.

However, based on the previous results and considerations it is reasonable to expect that the APC performance weakly depends on the specific multichannel processing approach.

\section{REFERENCES}

[1] G. Krieger, A. Moreira, H. Fiedler, I. Hajnsek, M. Werner, M. Younis, and M. Zink, "TanDEM-X: A satellite formation for high-resolution SAR interferometry," IEEE Trans. Geosci. Remote Sens., vol. 45, pt. 1, no. 11, pp. 3317-3341, Nov. 2007.

[2] W. Dierking, "Mapping of different sea ice regimes using images from Sentinel-1 and ALOS synthetic aperture radar," IEEE Trans. Geosci. Remote Sens., vol. 48, pt. 1, no. 3, pp. 1045-1058, Mar. 2010. 
[3] R. Werninghaus and S. Buckreuss, "The TerraSAR-X mission and system design," IEEE Trans. Geosci. Remote Sens., vol. 48, no. 2, pp. 606-614, Feb. 2010

[4] F. Caltagirone, G. De Luca, F. Covello, G. Marano, G. Angino, and M. Piemontese, "Status, results, potentiality and evolution of COSMO-SkyMed, the Italian Earth Observation constellation for risk management and security," in Proc. IEEE IGARSS, Honolulu, HI, Jul. 2010, pp. 4393-4396.

[5] T. Toutin, "Impact of RADARSAT-2 SAR ultrafine-mode parameters on stereo-radargrammetric DEMs," IEEE Trans. Geosci. Remote Sens., vol. 48 , no. 10 , pp. $3816-3823$, Oct. 2010.

[6] J. C. Curlander and R. N. McDonough, Synthetic Aperture Radar: Systems and Signal Processing. New York: Wiley, 1991

[7] G. Krieger, N. Gebert, and A. Moreira, "Multidimensional waveform encoding: A new digital beamforming technique for synthetic aperture radar remote sensing," IEEE Trans. Geosci. Remote Sens., vol. 46, no. 1, pp. 31-46, Jan. 2008

[8] G. Krieger, N. Gebert, M. Younis, and A. Moreira, "Advanced synthetic aperture radar based on digital beamforming and waveform diversity," in Proc. IEEE RadarCon, Rome, Italy, May 2008, pp. 767-772.

[9] M. Younis, F. Bordoni, N. Gebert, and G. Krieger, "Smart multi-aperture radar techniques for spaceborne remote sensing," in Proc. IEEE IGARSS, Boston, MA, Jul. 2008, vol. 3, pp. 278-281.

[10] G. Krieger, M. Younis, N. Gebert, S. Huber, F. Bordoni, A. Patyuchenko, and A. Moreira, "Advanced digital beamforming concepts for future SAR systems," in Proc. IEEE IGARSS, Honolulu, HI, Jul. 2010, pp. 245-248.

[11] A. Moreira, "Suppressing the azimuth ambiguities in synthetic aperture radar images," IEEE Trans. Geosci. Remote Sens., vol. 31, no. 4, pp. 885-895, Jul. 1993.

[12] J. Mittermayer and J. M. Martinez, "Analysis of range ambiguity suppression in SAR by up and down chirp modulation for point and distributed targets," in Proc. IEEE IGARSS, Tokyo, Japan, Jul. 2001, vol. 6, pp. 4077-4079.

[13] J. Dall and A. Kusk, "Azimuth phase coding for range ambiguity suppression in SAR," in Proc. IEEE IGARSS, Anchorage, AK, Sep. 2004, vol. 3, pp. 1734-1737.

[14] M. Suess, B. Grafmueller, and R. Zahn, "A novel high resolution, wide swath SAR system," in Proc. IEEE IGARSS, Sydney, Australia, Jul. 2001, vol. 3, pp. 1013-1015.

[15] D. Cristallini, M. Sedehi, and P. Lombardo, "Imaging solution based on azimuth phase coding," in Proc. EUSAR, Friedrichshafen, Germany, Jun. 2008, pp. 57-60.

[16] G. Krieger, N. Gebert, and A. Moreira, "Unambiguous SAR signal reconstruction from nonuniform displaced phase center sampling," IEEE Geosci. Remote Sens. Lett., vol. 1, no. 4, pp. 260-264, Oct. 2004.

[17] N. Gebert, G. Krieger, and A. Moreira, "Digital beamforming on receive: Techniques and optimization strategies for high-resolution wideswath SAR imaging," IEEE Trans. Aerosp. Electron. Syst., vol. 45, no. 2 , pp. 564-592, Apr. 2009

[18] S. Huber, M. Younis, A. Patyuchenko, and G. Krieger, "Digital beam forming techniques for spaceborne reflector SAR systems," in Proc. EUSAR, Aachen, Germany, Jun. 2010, pp. 962-965.

[19] F. Bordoni, M. Younis, N. Gebert, G. Krieger, and C. Fischer, "Performance investigation on the high-resolution wide-swath SAR system with monostatic architecture," in Proc. EUSAR, Aachen, Germany, Jun. 2010, pp. $1122-1125$.

[20] M. Younis, A. Patyuchenko, S. Huber, G. Krieger, and A. Moreira, "A concept for a high performance reflector-based X-band SAR," in Proc. EUSAR, Aachen, Germany, Jun. 2010, pp. 740-743.

[21] U. Steinbrecher, D. Schulze, J. Boer, and J. Mittermayer, "TerraSAR-X instrument operations rooted in the system engineering and calibration project," IEEE Trans. Geosci. Remote Sens., vol. 48, no. 2, pp. 633-641, Feb. 2010

[22] N. Gebert, "Multi-channel azimuth processing for high-resolution wide-swath SAR imaging," Ph.D. dissertation Universität Karlsruhe (TH), DLR-Forschungsbericht, Wessling, Germany, Jun. 2009, DLR-FB 2009-13.

[23] C. Fischer, C. Heer, G. Krieger, and R. Werninghaus, "A high resolution wide swath SAR," in Proc. EUSAR, Dresden, Germany, May 2006.

[24] C. Schaefer, M. Younis, and M. Ludwig, "Advanced SAR instrument based on digital beam forming," in Proc. Adv. RF Sens. Remote Sens. Instruments, Noordwijk, The Netherlands, Nov. 2009.

[25] A. Patyuchenko, M. Younis, S. Huber, and G. Krieger, "Optimization aspects of the reflector antenna for the digital beam-forming SAR system," in Proc. EUSAR, Aachen, Germany, Jun. 2010, pp. 954-957.

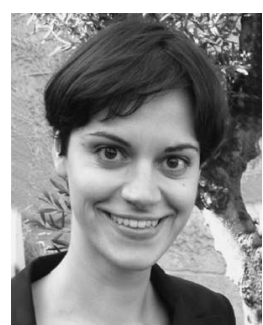

Federica Bordoni received the Laurea degree in telecommunication engineering and the $\mathrm{Ph} . \mathrm{D}$. degree in information engineering from the University of Pisa, Pisa, Italy, in 2001 and 2005, respectively.

From 2002 through 2004, she was with the Department of Ingegneria dell'Informazione of the University of Pisa, where her research activity included modern spectral estimation and detection techniques for multichannel and multidata signal processing, with applications both in the field of synthetic aperture radar (SAR) interferometry and radar surveillance. From May 2005 to July 2007 she worked at Alenia Space, Rome, Italy, on the validation/certification of COSMO-SkyMed SAR products. Since October 2007, she has been with the Microwaves and Radar Institute of the German Aerospace Center (DLR), Oberpfaffenhofen, Germany, where her activity has been focusing on the performance analysis and design of innovative multichannel SAR systems for high-resolution wide-swath imaging.

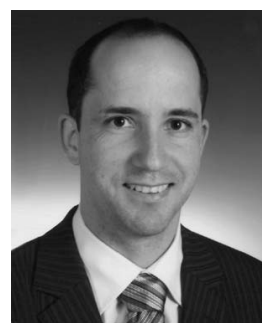

Marwan Younis (S'95-M'05-SM'08) was born in Las Cruces, in 1970. He received his B.Sc. degree in electrical engineering from the University of Baghdad, Baghdad, Iraq, in 1992 and the Dipl.-Ing. (M.Sc.) and Dr.-Ing. (Ph.D.) degrees in electrical engineering from the Universität Karlsruhe (TH), Karlsruhe, Germany, in 1997 and 2004, respectively.

From 1998 to 2004, he was a research scientist with the Institut für Höchstfrequenztechnik und Elektronik, Universität Karlsruhe (TH). Since 2005, he has been with the Microwaves and Radar Institute of the German Aerospace Center (DLR), Oberpfaffenhofen, Germany. He is the author and coauthor of over 70 conference papers and more than 15 reviewed publications. His research fields include synthetic aperture radar (SAR) systems, digital beamforming for radar, synchronization of bistatic SAR, forward looking radar, and antennas.

Dr. Younis is an active member of the IEEE and the German Association for Location and Navigation (DGON). He is the active microwave working group leader within the IEEE instruments and future technologies technical committee. He is a lecturer with the Universität Karlsruhe (TH). He received the Hermann-Billing award for his Ph.D. thesis in 2005.

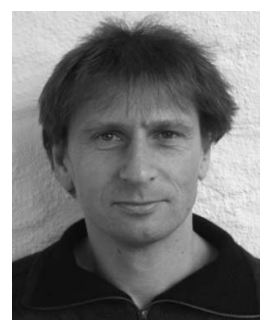

Gerhard Krieger (M'04-SM'10) received the Dipl.-Ing. (M.S.) and Dr.-Ing. (Ph.D.) degrees (with honors) in electrical and communication engineering from the Technical University of Munich, Munchen, Germany, in 1992 and 1999, respectively.

From 1992 to 1999, he was with the LudwigMaximilians University, Munich, where he conducted multidisciplinary research on neuronal modeling and nonlinear information processing in biological and technical vision systems. In 1999, he joined the Microwaves and Radar Institute (HR) of the German Aerospace Center (DLR), Oberpfaffenhofen, Germany, where he developed signal and image processing algorithms for a novel forward looking radar system employing digital beamforming on receive. From 2001 to 2007, he led the New SAR Missions Group which pioneered the development of advanced bistatic and multistatic radar systems as exemplified by the forthcoming TanDEM-X mission as well as innovative multichannel SAR techniques and algorithms for high-resolution wide-swath SAR imaging. Since 2008, he has been Head of the new Radar Concepts Department of the Microwaves and Radar Institute, DLR, Oberpfaffenhofen, Germany.

Dr. Krieger received several national and international awards, including the IEEE Transactions Prize Paper Award of the Geoscience and Remote Sensing Society and the Otto Lilienthal Sabbatical of the German Aerospace Center, DLR. His current research interests focus on the development of multichannel radar techniques and algorithms for innovative MIMO SAR systems, the demonstration of novel interferometric and tomographic earth observation applications, and the conceptual design of advanced bi- and multistatic radar missions. 